\title{
BMJ Open Management of recurrent pancreatic cancer after surgical resection: a protocol for systematic review, evidence mapping and meta-analysis
}

\author{
Jong-chan Lee, ${ }^{1}$ Soyeon Ahn, ${ }^{2}$ In Kuk Cho, ${ }^{1}$ Jongchan Lee, ${ }^{1}$ Jaihwan Kim, ${ }^{1}$
} Jin-Hyeok Hwang ${ }^{1}$

To cite: Lee J, Ahn S, Cho IK, et al. Management of recurrent pancreatic cancer after surgical resection: a protocol for systematic review, evidence mapping and meta-analysis. BMJ Open 2018;8:e017249. doi:10.1136/ bmjopen-2017-017249

- Prepublication history and additional material for this paper are available online. To view these files, please visit the journal online (http://dx.doi org/10.1136/bmjopen-2017017249).

Received 11 April 2017 Revised 16 February 2018 Accepted 23 February 2018

\section{Check for updates}

${ }^{1}$ Department of Internal Medicine, Seoul National University Bundang Hospital, Seongnam, Korea

${ }^{2}$ Department of Biostatistics, Seoul National University College of Medicine, Seoul National University Bundang Hospital, Seongnam, Korea

Correspondence to Dr Jin-Hyeok Hwang; woltoong@snu.ac.kr

\section{ABSTRACT}

Introduction Although recurrence rate among cases of resected pancreatic cancer are as high as $85 \%$, an optimal treatment for recurrent pancreatic cancer (RePC) has not been established. Previous evidence regarding RePC is scarce, and randomised controlled trials (RCTs) are particularly lacking. The evidence mapping (EM) method has been introduced as a tool intended to complement the conventional systematic review (SR) and meta-analysis (MA) and is suitable for this issue. This review aims to investigate the optimal treatment options for RePC, using a newly developed automatic EM tool.

Method and analysis All study types, including RCTs, non-randomised studies and other forms of observational studies will be included in the SR-EM. The Medline, Embase, Cochrane library and Scopus databases will be searched for reports of five treatment options for local and metastatic recurrences, including re-resection, chemotherapy, radiotherapy, best supportive care and other novel treatments, published from database inception to 30 April 2017. References from relevant studies will be searched manually. If meta-analysis is feasible, the primary outcome measure will be median overall survival. Two independent authors will select the studies and assess the risk of bias, and a third author will resolve discrepancies in consensus meeting. To visualise EM, we will use a novel web-based and open-access mapping programme, Plotting E-Map (PLOEM) (http://plotting-emap.com). If eligible combinations of interventions for quantitative comparison are identified, we will conduct subgroup MAs using random-effect models and $\mathrm{I}^{2}$ statistics. Publication bias will be visualised using funnel plots.

Ethics and dissemination This study will not use primary data, and therefore formal ethical approval is not required. The findings will be disseminated through peer-reviewed journals and committee conferences.

PROSPEROregistration number CRD42016049178.

\section{INTRODUCTION}

\section{Description of condition}

Pancreatic cancer is the fourth and fifth leading cause of cancer-related deaths in the USA and Europe, respectively. ${ }^{12}$ The 5-year

\section{Strengths and limitations of this study}

- This is the first systemic review of treatments for recurrent pancreatic cancer after surgical resection.

- To perform evidence mapping (EM) for multiple treatment options, we established a novel automatic web-based programme, Plotting E-Map (PLOEM) (www.plotting-e-map.com).

- The scope of this study encompasses both local and metastatic recurrences and five types of treatment: re-resection, chemotherapy, radiotherapy, best supportive care and other novel treatments.

- We distinct the concepts of 'evidence gap map' and 'EM'.

- Our study is limited by the lack of a well-designed randomised controlled trial of this condition.

survival rate of pancreatic cancer remains near $7 \%$ and has not significantly improved in several decades. ${ }^{12}$ As pancreatic cancer is difficult to detect in early stages, only approximately $20 \%$ of patients with pancreatic cancer are candidates for curative resection during the first diagnostic phase. ${ }^{3}$ Even more unfortunately, the recurrence rate of pancreatic cancer is as high as $85 \%$, even after curative resection. ${ }^{45}$

Recurrent pancreatic cancers (RePCs) manifest in one of two patterns: locoregional or metastatic recurrence. ${ }^{6}$ One retrospective observational study reported that the rates of local, metastatic and synchronous local/ metastatic RePCs were $17 \%, 60 \%$ and $23 \%$, respectively. ${ }^{7}$ Although the median overall survival (OS) after the first resection was 18.7 months in that study, the recurrence patterns did not affect OS. We note that the metastatic recurrence rate was higher than the local recurrence rate in that study; however, our pilot search found that treatment evidences for metastatic RePC were scarcer than for local RePC. 


\begin{tabular}{ll}
\hline Table 1 & Abbreviations \\
\hline Abbreviations* & Full terms \\
\hline BSC & Best supportive care \\
CRT & Chemoradiation therapy \\
DEF & Data extraction form \\
EGM & Evidence gap map \\
EM & Evidence mapping \\
MA & Meta-analysis \\
ONT & Other novel treatment \\
OS & Overall survival \\
PLOEM & Plotting evidence map \\
RCT & Randomised-controlled trial \\
ReOP & Reoperation (ie, re-resection) \\
RePC & Recurrent pancreatic cancer \\
SC & Supportive care \\
SR & Systematic review \\
\hline
\end{tabular}

${ }^{*}$ In alphabetical order.

\section{Description of the intervention}

According to our pilot search, RePC treatment options could be categorised into five groups: re-resection (ReOP), systemic chemotherapy, radiation therapy, best supportive care (BSC) and other novel treatments (ONT). The abbreviations are listed in table 1 .

ReOP is defined as the surgical resection of a recurrent pancreatic cancer mass for curative purposes, with or without subsequent chemotherapy, and includes locally recurred tumourectomy, total pancreatectomy, metastatectomy and combinations of these operations. In 1975, Ellis et al first reported the second-look operation for RePC, a palliative gastrojejunostomy for gastric outlet obstruction but not a curative ReOP. ${ }^{8}$ The first retrospective study of curative ReOP was performed by Nakeeb et $a l$ in 1995 and included 27 patients who underwent pancreaticoduodenectomy. ${ }^{9}$ Since then, studies of ReOP with various designs have been conducted, ${ }^{10-12}$ but no randomised controlled trials (RCTs) had been published at the time of our pilot screening.

Chemotherapy indicates systematic chemotherapy for local or metastatic RePC without curative ReOP or concurrent chemoradiation therapy. Since the first report of quadruple chemotherapy in recurrent gastrointestinal carcinoma by Cheng et al in $1977,{ }^{13}$ studies of RePC in diverse settings have been introduced and have mainly addressed gemcitabine-based regimens. ${ }^{14-16}$ Although several abstracts from medical conferences and small studies reported the use of other regimens, such as modified FOLFIRINOX or monoclonal antibodies, for RePC, ${ }^{17} 18$ few RCTs have used regimens other than gemcitabine according to our pilot search.

Radiotherapy includes both 'radiation only' and chemoradiation therapy (CRT, also called concurrent chemoradiation therapy) with a curative aim. Although most curative radiation treatments for pancreatic cancer involve CRT, in which the chemotherapeutic agent is used for radiosensitisation, ${ }^{19}$ we also include "radiation only' treatment because early studies (ie, before 1990s) reported radiotherapy without chemotherapeutic agents. $^{2021}$ From a technical viewpoint, radiotherapy includes conventional external beam radiation therapy, stereotactic body radiation therapy, intensity-modulated radiation therapy and intraoperative radiotherapy. ${ }^{22}$

Unlike other treatment options, BSC has been inconsistently defined among various clinical trials and has been used interchangeably with 'supportive care (SC) '. ${ }^{23}$ Whereas the definition of 'palliative care' is relatively homogenous, ${ }^{25} 26$ the definitions of BSC vary widely. ${ }^{27}$ Because the present review focuses on 'survival' rather than on the BSC modality itself, we will not use a strict or narrow definition of BSC. Rather, we will accept each original article's definition of BSC/SC and will tabulate each definition in the online supplementary materials.

ONT is another highly heterogeneous RePC treatment category. It includes all treatment options not included in the ReOP, chemotherapy, radiotherapy and BSC categories. According to our pilot search, ONT includes cryoablation, vaccine therapy, partial pancreatic autotransplantation and proton therapy. ${ }^{28-31}$ These heterogeneous treatment modalities could not be classified into a single group in a conventional meta-analysis (MA). However, the present study will use an 'evidence mapping' (EM) tool, thus allowing us to 'map' time trends or detailed information about these various modalities. ${ }^{32}$ We will include this ONT group only for mapping and not for quantitative analysis.

\section{Controversy regarding treatment of RePC}

Clinically, recent European (European Society for Medical Oncology) and US (American Society of Clinical Oncology) guidelines did not include treatment recommendations for RePC. ${ }^{33-36}$ Only the US National Comprehensive Cancer Network (NCCN) guideline suggests multiple treatment options, such as clinical trials (CRT) (for CRT-naive cases), systemic chemotherapy or BSC, for RePC patients. ${ }^{6}$ The current NCCN guideline states that for selected cases with a good performance status and favourable tumour location, surgical resection may be considered but is not recommended due to lack of evidence. ${ }^{6}$ Only one study of pulmonary metastatectomy, by Arnaoutakis et al, reported a survival gain in patients with isolated lung metastases of pancreatic cancer, ${ }^{37}$ and this is not firm evidence.

Methodologically, it is very difficult to evaluate the small amount of scattered evidence regarding issues related to RePC when using conventional systematic review (SR) and MA methods. Moreover, a networking process is needed because more than two treatment modalities exist. EM could be useful in this setting; however, no tool for EM has been established or standardised. ${ }^{32}$ In summary, the following major questions regarding the treatment of RePC have been raised: (1) which treatment modality is 
optimal for RePC patients and (2) how can we evaluate the existing scattered, limited and multiarmed evidence using EM.

\section{Importance of this work}

First, this is the initial attempt to 'map' entire types of evidence regarding the treatment of RePC, from case reports to RCTs. When sufficient bodies of evidence exist, such as double-armed RCTs, conventional SR and MA methods may be able to provide quantitative conclusions. However, an alternative or complementary modality to SR is needed for settings that include multiple, heterogeneous, small-sized and scattered bodies of evidence. We will therefore adopt the EM method to perform this task. We expect that our work will help both clinicians who facing clinical decisions regarding RePC and researchers who plan to conduct prospective trials of RePC treatment.

Second, we established a novel EM tool, Plotting E-map (PLOEM), which has already been launched on an open-access website ${ }^{38}$ and disseminated at an international conference during an oral presentation. ${ }^{39}$ Using this automatic device, users can easily visualise the evidence related to their issue of interest, including the study types, study numbers, time factors and all options for multiple interventions. We expect that this open-access device will help researchers planning EM for any type of medical or public health issue. We will examine PLOEM in detail in the Discussion section.

Third, if possible, we will conduct MAs of the eligible combinations of treatment options as subgroup analyses. If existing unfounded evidence can be pooled into an analysis, this review will provide quantitative evidence using subgroup MAs. As decisions regarding the ability to perform MAs will be made after the final EM process, this study may be described as a SR-EM or SR-EM-MA during the main article phase. Accordingly, we have set the title as SR-EM-MA during this protocol phase.

\section{OBJECTIVES \\ Main objectives}

This SR-EM-MA aims to visualise and structuralise the trends and efficacies of the five treatment options, including ReOP, chemotherapy, radiotherapy, BSC and ONT for RePC after curative resection, using the novel automatic mapping device PLOEM.

\section{Secondary objectives}

1. To develop and launch a novel EM device, PLOEM V.2.0;

2. To explore the trends of RePC treatment by several era, based on important points including introductions of new chemotherapeutic regimens or new OP techniques;

3. If possible, to conduct the meta-analyses or network meta-analyses in the eligible comparative studies after checking the feasibility of the quantitative meta-analyses.

\section{METHODS AND ANALYSIS}

Because this study is an SR, it will be performed according to the recommendations of the Preferred Reporting Items for Systematic Reviews and Meta-Analyses statement
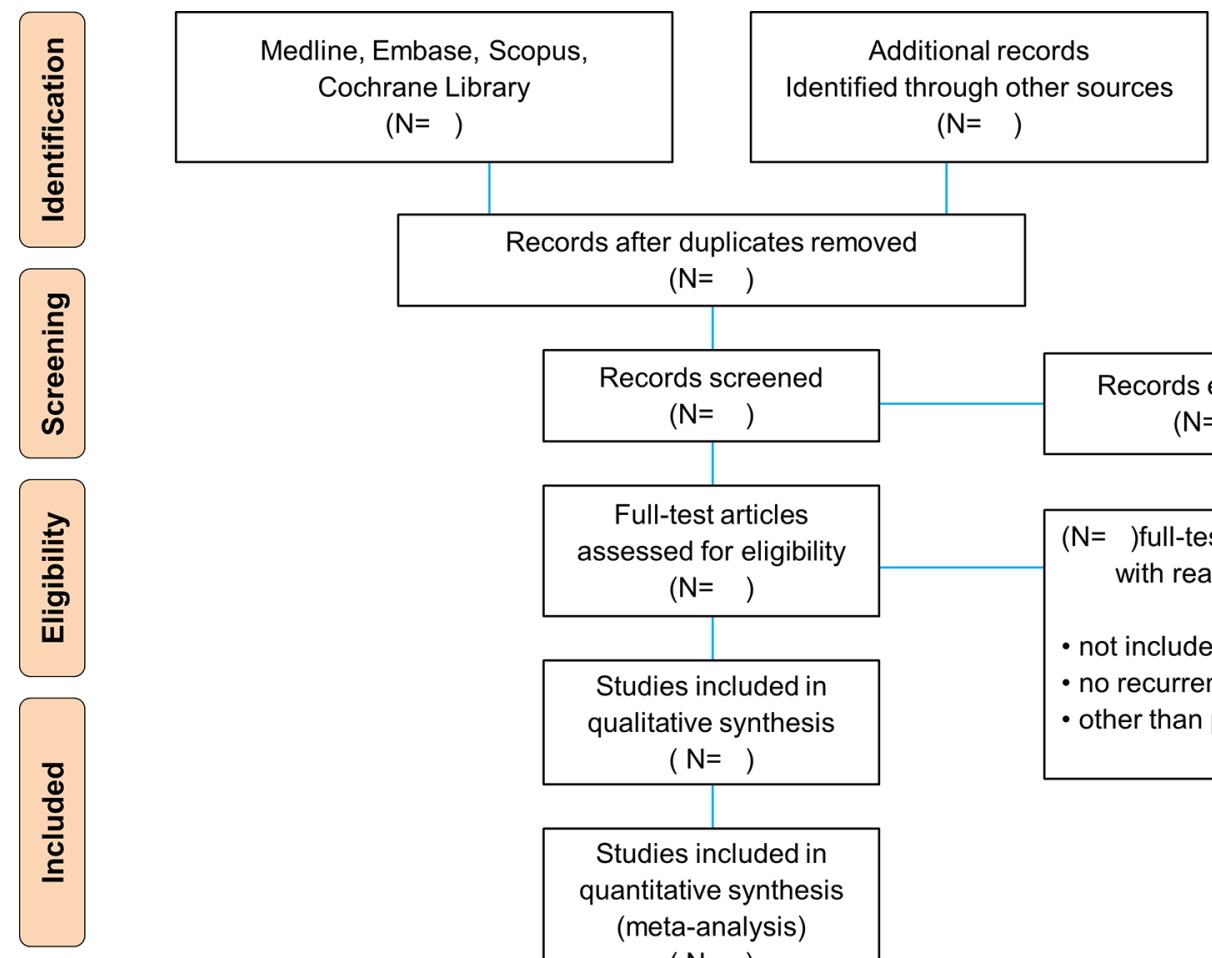

Records after duplicates removed $(\mathrm{N}=)$

\begin{tabular}{|c|c|}
\hline $\begin{array}{l}\text { Records screened } \\
\qquad(\mathrm{N}=)\end{array}$ & $\begin{array}{l}\text { Records excluded } \\
\qquad(\mathrm{N}=)\end{array}$ \\
\hline $\begin{array}{l}\text { Full-test articles } \\
\text { assessed for eligibility } \\
\qquad(\mathrm{N}=)\end{array}$ & $\begin{array}{l}(\mathrm{N}=) \text { full-test articles escluded } \\
\text { with reasons: }\end{array}$ \\
\hline $\begin{array}{l}\text { Studies included in } \\
\text { qualitative synthesis } \\
\qquad(\mathrm{N}=)\end{array}$ & $\begin{array}{l}\text { - not included survival data } \\
\text { - no recurrence data } \\
\text { - other than pacreatic cancer }\end{array}$ \\
\hline
\end{tabular}

Studies included in quantitative synthesis (meta-analysis) $(\mathrm{N}=)$

Figure 1 PRISMA flowchart. PRISMA, Preferred Reporting Items for Systematic Reviews and Meta-Analyses . 
Table 2 Study types

\begin{tabular}{|c|c|c|c|c|c|c|c|c|}
\hline \multirow[b]{4}{*}{ Character } & \multicolumn{4}{|c|}{ Observational } & \multicolumn{4}{|l|}{ Experimental } \\
\hline & \multicolumn{4}{|c|}{ Single-armed study } & \multicolumn{4}{|c|}{ Double-armed study } \\
\hline & \multicolumn{3}{|l|}{ Retro } & \multirow{2}{*}{$\begin{array}{l}\text { Pro } \\
\text { D } \\
\end{array}$} & \multirow{2}{*}{$\begin{array}{l}\text { Retro } \\
\text { E }\end{array}$} & \multicolumn{3}{|l|}{ Pro } \\
\hline & A & B & $\mathbf{C}$ & & & $\mathbf{F}$ & G & $\mathbf{H}$ \\
\hline Study types & $\begin{array}{l}\text { Case } \\
\text { report }\end{array}$ & $\begin{array}{l}\text { Case } \\
\text { series }\end{array}$ & $\begin{array}{l}\text { Single-armed } \\
\text { retrospective } \\
\text { study }\end{array}$ & $\begin{array}{l}\text { Single-armed } \\
\text { prospective } \\
\text { study }\end{array}$ & $\begin{array}{l}\text { Double- } \\
\text { armed } \\
\text { retrospective } \\
\text { study }\end{array}$ & $\begin{array}{l}\text { Double- } \\
\text { armed } \\
\text { prospective } \\
\text { study }\end{array}$ & $\begin{array}{l}\text { Double- } \\
\text { armed } \\
\text { experimental } \\
\text { study (NRS) }\end{array}$ & $\mathrm{RCT}$ \\
\hline \multicolumn{9}{|l|}{ Dot design } \\
\hline Included in EM & Yes & Yes & Yes & Yes & Yes & Yes & Yes & Yes \\
\hline Included in MA & No & No & If possible & If possible & If possible & If possible & If possible & If possible \\
\hline Assessment & $\mathrm{NIH}^{50}$ & $\mathrm{NIH}^{50}$ & $\mathrm{Hoy}^{49}$ & $\mathrm{Hoy}^{49}$ & ROBINS-1 ${ }^{48}$ & ROBINS-1 $\left.\right|^{48}$ & ROBINS-1 $1^{48}$ & $\mathrm{RoB}^{47}$ \\
\hline GRADEpro & No & No & Yes & Yes & Yes & Yes & Yes & Yes \\
\hline
\end{tabular}

EM, evidence mapping; MA, meta-anaylsis; NIH, National Institutes of Health; NRS, non-randomised study; RCT, randomised controlled trial; RoB, risk of bias; ROBINS-I, risk of bias in non-randomised studies of interventions.

(figure 1). ${ }^{40}$ However, no official standard methodology has been developed for an EM. ${ }^{32} 41-44$ Therefore, we aim to develop a new set of reporting items for EM. The details of these new items will be mentioned in the Discussion section. A review protocol for this study has been published in the International Prospective Register of Systematic Reviews under registration number CRD 42016049178.

\section{CRITERIA FOR STUDY CONSIDERATION IN THIS REVIEW Types of studies}

Because this SR primarily aimed to map trends, we will use the 'all times, all types' strategy. In this context, we will include almost all kinds of study designs such as case reports, case series, cross-sectional (descriptive and analytical) studies, case-control (retrospective and prospective, ie, nested case-control and case-cohort studies), cohort studies (retrospective and prospective) and clinical or experimental trials (randomised and non-randomised studies). To visualise and structuralise these designs intuitionally, we reclassified and summarised all the study types in table 2. To distinguish between case series and cohort studies, we will follow the definition of Methes $e t$ $a t^{45}$ and Dekkers $e t a l^{46}$

\section{Participants}

Participants in this review will be 18 years of age or older, diagnosed with RePC after curative surgical resection for primary pancreatic cancer. Because answers to the question of 'how many months after resection should be defined as a recurrence?' vary among the previous studies, we will not define recurrence according to a specific period of time. Instead, we will define a RePC as a new case of pancreatic cancer that was not identified during the first postoperative imaging exam. Pathologically, we will include only pancreatic ductal adenocarcinomas. We will classify RePC cases as local or metastatic recurrence. If both groups are included in a single study, these will also be pooled and analysed separately.

According to study design, we will not exclude any kinds of studies basically, because this review aims to comprehensively map all treatment options for RePC. If a study includes a diverse population and the ductal adenocarcinoma is only a part of the study, we will ask the author for the original data. If this is not feasible, we will include the study only in visual mapping (EM), but we will exclude it from quantitative analysis of efficacy or toxicity.

In terms of population composition, we will exclude following studies: studies for (1) patients with pancreatic tumours other than ductal adenocarcinoma, (2) patients who have previously undergone initial pancreatic surgery with a palliative or explorative rather than curative aim and (3) patients who had remnant pancreatic cancer after surgery or incomplete resection.

\section{Interventions and comparators}

Whereas conventional MAs compare one intervention with one control, in our study, each of the five treatment options serves as both an intervention and a control of each other.

ReOP includes only curative resection, which encompasses remnant tumourectomy, pancreatectomy for locoregional recurrence or metastatectomy for remote recurrence. Chemotherapy includes any type of regimen for pancreatic cancer treatment. Chemotherapy will be pooled and visualised into a mapping tool regardless of the type of regimen, but detailed information about each regimen will be presented in a supplementary table 1 .

Radiotherapy includes therapeutic radiation, with or without concurrent chemotherapy; accordingly, only palliative radiotherapy, such as pain control for bone metastases, will not be included in this treatment option. Instead, 
palliative radiotherapy without curative treatment will be classified as BSC. Within the radiotherapy category, CRT will include any type of chemotherapeutic agent and any radiation dose and will be pooled and visualised using a mapping tool. Detailed information about CRT will be presented in another online supplementary table.

BSC includes general conservative management and palliative treatments such as surgery for obstruction or bleeding and radiation for pain control. ONT includes any type of novel treatment option not classified in one of the former four categories, as discussed in the Introduction section. As the ONT group will inevitably include highly heterogeneous treatment modalities, we will only use this group to visualise trends and not for quantitative analysis. Detailed information about ONT will also be presented in separate online supplementary table.

In patients with multiple and serial treatment modality, the classification is according to the 'first' treatment option. For example, if one patient received (CRT $\rightarrow$ chemotherapy $\rightarrow \mathrm{BSC}$ ), he or she will be regarded as the CRT group.

\section{Outcome measures}

Because this review is basically an EM, the outcome measure is visualisation of mapping itself. However, to structuralise the detailed trends, we will explore treatment trends according to (1) use of treatment, (2) efficacy (overall survival) over time and (3) side effects of treatments.

If there are feasible studies for quantitative analysis, we will conduct the meta-analysis or network meta-analysis. In this case, the primary outcome will include OS. Given the characteristics of RePC, OS should be classified into survival after the first resection and survival after the first detection of recurrence. We will use these two concepts separately. If any study does not distinguish these survival concepts, we will contact the author to validate the survival definition. Regarding EM, the primary outcome is the treatment trend itself, which will be visualised using a graphically mapping tool.

\section{Data search and selection}

\section{Data sources}

Four electronic databases, Medline, Embase, Cochrane Library and Scopus, will be searched from their inception to March 2017. In addition, cited references from relevant articles and all abstracts from major international conferences will be manually searched.

\section{Search strategy}

The global search strategy is shown in table 3, and the practical search terms will be applied according to the query entry rules for individual databases. If any up-todate evidence is published during the review period, we will evaluate the eligibility of each study and consider its addition to the suitable group. Web of Science will be used to conduct a manual search for citations of relevant articles.

\section{Inclusion and exclusion criteria}

The screening and inclusion process will involve two steps: first, title and abstract; and second, a full-text review. Two independent authors ( $\mathrm{J}-\mathrm{cL}$ and $\mathrm{J}-\mathrm{HK}$ ) will conduct this process. Any discrepancies will be resolved in a consensus meeting with a third author (SA). We will use citation managers such as Endnote to create new folders that meet various conditions.

\section{Assessment of the risk of bias in included studies}

The risk of bias will be independently evaluated by two authors (J-cL, J-HK). The result of this assessment will be verified by a third author (SA). For RCTs, the 'risk of bias' tool in the Cochrane Handbook (V.5.1.0) will be used. ${ }^{47}$ For non-randomises studies (NRS) of interventions, we will use risk of bias in non-randomised studies of interventions tool ${ }^{48}$ For survival rates of single-arm studies, we will use the risk of bias in prevalence studies tool by Hoy et $a l .{ }^{49}$ For case reports and case series, the National Institutes of Health quality assessment tool will be used. ${ }^{50}$

\section{Evidence mapping and its novel tool, PLOEM}

In previous studies, the EM was sometimes, but not always, described as an evidence gap map (EGM).$^{41}{ }^{51-53}$ This could confuse the researchers and readers. We distinguished the EM from the EGM for the following reasons: (1) the EGM is similar to the alternative of SR and could be used as a bridgehead for subsequent research. The EM, on the other hand, is somewhat complementary to SR. (2) Both EM and EGM can focus on visualisation; however, EM weighs visual graphs, whereas EGM uses

Table 3 Global search strategy

\begin{tabular}{llll}
\hline Category & Pancreatic cancer & Recurrent & After surgical resection \\
\hline Search term & \#1. pancreatic & \#8. recurrent & \#13. resection \\
& \#2. pancreas & \#9. recurrence & \#14. operation \\
& \#3. cancer & \#10. recurred & \#15. surgery \\
& \#4. adenocarcinoma & \#11. recur & \#16. surgical \\
& \#5. neoplasm(s) & \#12. relapse & \\
\#6. tumour(s) & & \#18. (or/13-16) \\
\hline
\end{tabular}




\section{- Input page}

\section{"PLOEM}
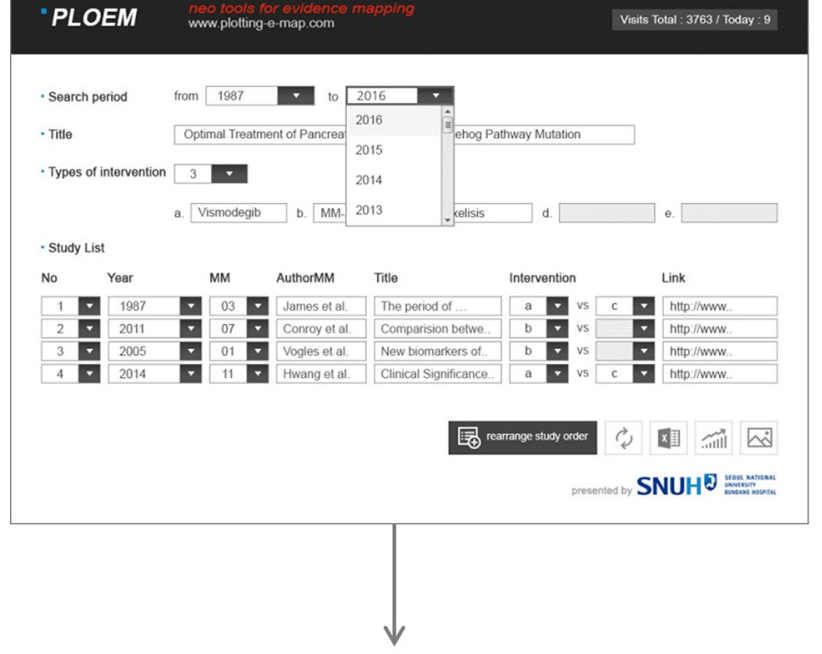

\section{- Output page}

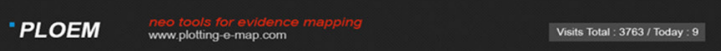

or

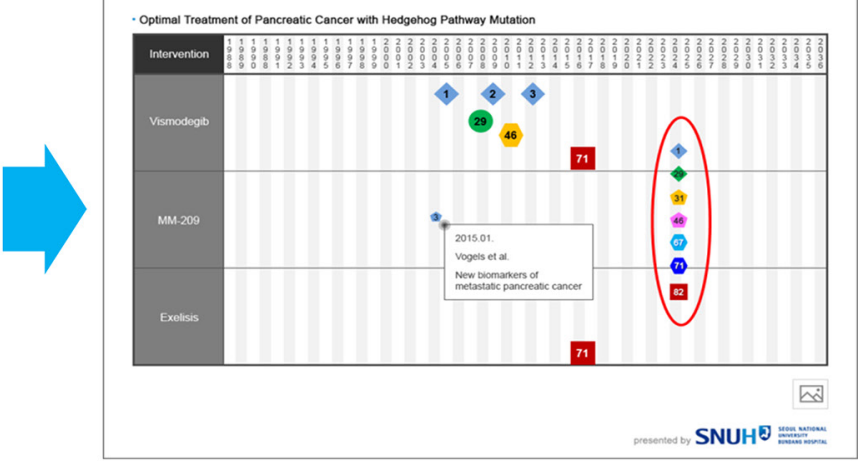

\section{- Data extract Form}

- download as excel file

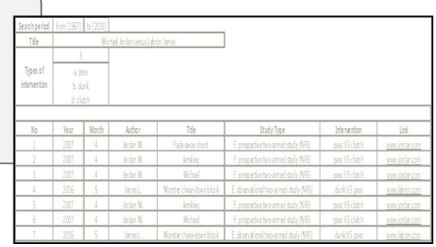

Figure 2 Basic structure of PLOEM V.1.0. PLOEM, Plotting E-Map.

visual tables. In this review, we will adopt EM rather than EGM.

To visualise multiple types of evidence easily and reproducibly, we developed a new mapping tool, PLOEM, using the Javascript language. This web-based, open-access and automatic visualising tool is intended for users performing EM. Unlike several earlier EM methods, ${ }^{41} 43$ PLOEM does not treat evidence gaps. Instead, it focuses on 'automatic mapping' itself when dealing with complex evidence. We have already launched a free website for PLOEM V.1.0 (http://www.plotting-e-map.com) and now developing PLOEM V.2.0. The upgrade items are shown in online supplementary table 1 .

The basic structure of PLOEM comprises two pages: the input and output pages (figure 2). On the input page, users can insert basic information about eligible studies in a line-by-line manner. Using the data extraction form (DEF) button on this page, users can directly download information entered into a Microsoft Excel file. After completing the input page, users can easily and automatically obtain the visualised results using the Graph button. On the output page, each dot shape depicts the type of study in table 2 . The number inside each dot matches the study identification (ID), and when clicked, each dot links to its corresponding study. Using the mouse rollover function, users can easily check the basic information about each dot in a pop-up box. On the output page, users can observe trends in evidence by time, treatment modality and study type.

\section{Data extraction}

If eligible studies are found to use specific combinations of treatments, two authors (J-cL, IC) will individually extract the data. The extraction process will be based on the DEF. The data extraction process will be established in consensus meeting (J-cL, IC and SA). We will extract data from each included study as follows:

- (1) Study characteristics: study ID, (2) reviewer name, (3) title, (4) author, (5) published year, (6) nation, (7) hospital level, (8) sample size and (9) study design;

- (1) Demographic characteristics: age (with median and range), (2) sex as percentage of females, (3) recurrence type, (4) recurrence-free period, (5) pathological margin results (such as R0, R1 and R2) and (6) outcomes of each study;

- (1) Check for exclusion: other than the original article, (2) duplication, (3) pathology other than adenocarcinoma, (4) incomplete surgery and (5) not including recurrence.

If the required data are ambiguous or not reported in the clinical articles, the authors will contact the first or corresponding author of the study by telephone or email and collect the missing data using the DEF.

\section{Statistical analysis}

All statistical syntheses and analyses will be performed using Review Manager Software V.5.3 (Cochrane Collaboration, http://tech.cochrane.org/RevMan). If more than two studies of each type are eligible for analysis, we will combine the studies for a meta-analysis by study type. Different 
study designs will be analysed separately. The meta-analysis portion could be limited.

\section{Effect size and pooled estimate (model)}

If eligible studies exist, we will synthesise the HRs of OS. If the information from an individual study is insufficient, we will estimate the overall HR using practical methods for incorporating the summary time-to-event data into a meta-analysis of RCTs. ${ }^{54}$ Although this review is conducted carefully, each group should have high heterogeneity inevitably for following reasons. First, the conditions of patients such as age, performance status, comorbidity are various and they influence the treatment modality. Second, even in the same metastatic recurrence group, the diseases burdens are various. We will use the DerSimonian-Laird random-effects model to obtain pooled estimates for RCTs and NRS, given the expected high heterogeneity in each group. ${ }^{55}$

\section{Heterogeneity analysis}

Based on the clinical characteristics of RePC and our pilot search, the heterogeneity among studies is expected to be very high. If possible, heterogeneity among studies will be evaluated using $\mathrm{I}^{2}$ statistics, with $30 \%, 50 \%$ and $75 \%$ as the cut-off points for low, moderate and high degrees of heterogeneity, respectively. ${ }^{56}$ We will perform another type of subgroup analysis by specific era, because the treatment paradigm has changed according to several important regimens such as FOLFIRINOX or nab-paclitaxel. The improvement of surgical techniques will also be considered as well as the improvement of chemotherapy.

\section{Publication bias}

If MA is possible, we will visualise the publication bias in each group using contour-enhanced funnel plots. ${ }^{57}$ However, because the present study emphasises EM, the publication bias analysis will be optional.

\section{Evaluation of the level of evidence}

The level of evidence will be evaluated using the Grading of Recommendations Assessment, Development and Evaluation (GRADE) system and the GRADEpro GDT programme (GRADEpro Guideline Development Tool working group, https://gradepro.org/). ${ }^{58}$ These tables will include a summary of the intervention effect and the quality of individual outcomes, using the GRADE approach. The quality of the body of evidence for each outcome will be assessed based on five factors: study limitations, effect consistency, imprecision, indirectness and publication bias.

\section{DISCUSSION}

This study will provide two important perspectives: (1) clinical implications and (2) methodological implications.

First, regarding the clinical viewpoint, no consensus has been reached in terms of the management and treatment guidelines for RePC, particularly for surgical intervention. However, in actual practice, our centre often performs ReOPs for solitary metastatic recurrences, and our pilot study of the reports from surgical resection demonstrated a 'steady-seller' pattern. This provided the first motivation for this study.

Second, no standardised tools for EM have been developed, in contrast to RevMan for conventional MAs or WinBUGS for Bayesian MAs. ${ }^{32} 5960$ Accordingly, researchers conducting EM have been required to draw graphics manually, without assistance from automatic tools. Moreover, no available tools could simultaneously demonstrate time factors, multiple treatments, study types and comparisons. In this context, we established a novel device for EM. We expect some criticism of EM as a mere 'mapping' method with no quantitative analysis. However, many issues meet the following parameters: (1) clinical importance, (2) insufficient RCT data, (3) evidence scattered among multiple databases and abstracts and (4) multiple treatment options.

In conclusion, this review will provide new SR-EM-MA of the available treatments and research for RePC, and we expect that our study results will be applied to the updated guidelines for pancreatic cancer. From a methodological viewpoint, this study will serve as a powerful and standardised tool for EM.

\section{Ethics and dissemination}

This SR-EM-MA does not require formal ethical approval because the data used in this analysis do not involve personal information and thus do not affect privacy. The findings of this proposed SR-EM-MA will provide a general overview and evidence of management of RePC with respect to OS. The findings will be disseminated through peer-reviewed publications or conference presentations.

Acknowledgements The authors thank Hyun Jung Kim, Professor of Korea Medical University for the guidance of search strategy. The authors also thank Editage for English proofreading. The authors thank Vizen Software for executive programming of PLOEM V.1.0. The authors thank Jinwook Seo and Brian J Lee in the Department of Computer Science and Engineering in Seoul National University for updating PLOEM V.2.0.

Contributors J-CL and J-HH planned the protocol. SA assisted with protocol design. JK provided clinical advice regarding the study protocol. JL revised the search strategy. J-CL, SA and J-HH drafted the protocol. JL and IKC search for studies and extract and analyse data. SA played a critical role in reviewing NRS and J-CL conceptualised and designed the PLOEM V.1.0.

Funding This research received no specific grant from any funding agency in the public, commercial or not-for-profit sectors.

Competing interests None declared.

Patient consent Not required.

Provenance and peer review Not commissioned; externally peer reviewed.

Open Access This is an Open Access article distributed in accordance with the Creative Commons Attribution Non Commercial (CC BY-NC 4.0) license, which permits others to distribute, remix, adapt, build upon this work non-commercially, and license their derivative works on different terms, provided the original work is properly cited and the use is non-commercial. See: http://creativecommons.org/ licenses/by-nc/4.0/

(C) Article author(s) (or their employer(s) unless otherwise stated in the text of the article) 2018. All rights reserved. No commercial use is permitted unless otherwise expressly granted.

\section{REFERENCES}

1. Siegel RL, Miller KD, Jemal A, et al. Cancer statistics, 2015. CA Cancer J Clin 2015;65:5-29. 
2. De Angelis R, Sant M, Coleman MP, et al. Cancer survival in Europe 1999-2007 by country and age: results of EUROCARE-5-a population-based study. Lancet Oncol 2014;15:23-34.

3. Rahib L, Smith BD, Aizenberg R, et al. Projecting cancer incidence and deaths to 2030: the unexpected burden of thyroid, liver, and pancreas cancers in the United States. Cancer Res 2014;74:2913-21.

4. Oettle H, Neuhaus P, Hochhaus A, et al. Adjuvant chemotherapy with gemcitabine and long-term outcomes among patients with resected pancreatic cancer: the CONKO-001 randomized trial. JAMA 2013;310:1473-81.

5. Schnelldorfer T, Ware AL, Sarr MG, et al. Long-term survival after pancreatoduodenectomy for pancreatic adenocarcinoma: is cure possible? Ann Surg 2008;247:456-62.

6. National Comprehensive Cancer Network (NCCN). Pancreatic adenocarcinoma; version 2. 2017. http://www.nccn.org/ professionals/physician gls/pdf/pancreatic.pdf

7. Van den broeck A, Sergeant G, Ectors N, et al. Patterns of recurrence after curative resection of pancreatic ductal adenocarcinoma. Eur $J$ Surg Oncol 2009;35:600-4.

8. Ellis H. Is a "second look operation" justified in suspected recurrences after abdominal cancer surgery? Br J Surg 1975;62:830-2.

9. Nakeeb A, Lillemoe KD, Cameron JL. The role of pancreaticoduodenectomy for locally recurrent or metastatic carcinoma to the periampullary region. J Am Coll Surg 1995;180:188-92.

10. Takamatsu S, Ban D, Irie T, et al. Resection of a cancer developing in the remnant pancreas after a pancreaticoduodenectomy for pancreas head cancer. J Gastrointest Surg 2005;9:263-9.

11. Thomas RM, Truty MJ, Nogueras-Gonzalez GM, et al. Selective reoperation for locally recurrent or metastatic pancreatic ductal adenocarcinoma following primary pancreatic resection. J Gastrointest Surg 2012;16:1696-704.

12. Strobel O, Hartwig W, Hackert T, et al. Re-resection for isolated local recurrence of pancreatic cancer is feasible, safe, and associated with encouraging survival. Ann Surg Oncol 2013;20:964-72.

13. Cheng FC, Lee CM, Ong GB. Quadruple chemotherapy in the treatment of advanced or recurrent gastrointestinal carcinoma. Clin Oncol 1977:3:191-5.

14. Sato Y, Tohma H, Shikama T. Chemotherapy for advanced and recurrent cancer patients - the effect of combination chemotherapy using cisplatin, peplomycin, mitomycin C, adriamycin, and 5-fluorouracil. Nihon Gan Chiryo Gakkai Shi 1990;25:1565-70.

15. Horiuchi $\mathrm{H}$, Uchida S, Hisaka T, et al. A study of recurrent pancreatic cancer with metastatic liver tumors after pancreatectomy. Gan To Kagaku Ryoho 2005;32:1685-7.

16. Taniyama TK, Morizane C, Nakachi K, et al. Treatment outcome for systemic chemotherapy for recurrent pancreatic cancer after postoperative adjuvant chemotherapy. Pancreatology 2012;12:428-33.

17. Vivaldi C, Vasile E, Fornaro L, et al. 2362 Second-line treatment after disease progression following first-line chemotherapy with modified FOLFIRINOX in advanced pancreatic cancer patients. Eur $\mathrm{J}$ Cancer 2015;51:S456-7.

18. Arlen M, Wang X, Luka J, et al. The use of specific monoclonal antibodies to target immunogenic tumor membrane proteins in patients with recurrent pancreatic and colon cancer. Curr Drug Deliv 2012;9:52-6.

19. Vincent A, Herman J, Schulick R, et al. Pancreatic cancer. The Lancet 2011:378:607-20.

20. Yamada S, Ishibashi T, Sasauchi S, et al. A case of recurrent pancreatic carcinoma treated by low-dose rate telecobalt therapy. Gan No Rinsho 1984;30:75-80.

21. Latz D1, Schraube P, Eble MJ. The primary radiotherapy of inoperable or recurrent pancreatic carcinoma-Heidelberg patients from 1982 to 1992. Strahlenther Onkol 1993;169:387-93.

22. Pazdur R, Wagman LD, Camphausem KA. Cancer management: a multidisciplinary approach: medical, surgical \& raditation oncology.11th Edn: CMPmedica, 2008:978.

23. Nipp RD, Currow DC, Cherny NI, et al. Best supportive care in clinical trials: review of the inconsistency in control arm design. $\mathrm{Br} \mathrm{J}$ Cancer 2015;113:6-11.

24. Hui D, Mori M, Parsons HA, et al. The lack of standard definitions in the supportive and palliative oncology literature. J Pain Symptom Manage 2012;43:582-92.

25. World Health Organization. WHO definition of palliative care. 2012. http://www.who.int/cancer/palliative/definition/en/

26. O'Connor M. Mission statements: an example of exclusive language in palliative care? Int J Palliat Nurs 2005;11:190-5.
27. Hui D, De La Cruz M, Mori M, et al. Concepts and definitions for "supportive care," "best supportive care," "palliative care," and "hospice care" in the published literature, dictionaries, and textbooks. Support Care Cancer 2013;21:659-85.

28. Pusceddu C, Melis L, Sotgia B, et al. Computed tomography-guided cryoablation of local recurrence after primary resection of pancreatic adenocarcinoma. Clin Pract 2015;5:741.

29. Tsujitani S, Tanii M, Yonemitsu Y. Clinical evaluation of dendritic cell based vaccines pulsed with WT1 and/or MUC1 for patients with advanced or recurrent cancers. ESMO Congress 2012.

30. Kobayashi T, Sato Y, Hirukawa H, et al. Total pancreatectomy combined with partial pancreas autotransplantation for recurrent pancreatic cancer: a case report. Transplant Proc 2012;44:1176-9.

31. Plastaras JP, Berman A, Apisarnthanarax $S$, et al. Proton reirradiation of locally recurrent pancreatic and ampullary adenocarcinomas. ASCO Gl cancer symposium 2013.

32. Miake-Lye IM, Hempel S, Shanman R, et al. What is an evidence map? A systematic review of published evidence maps and their definitions, methods, and products. Syst Rev 2016;5:28.

33. Khorana AA, Mangu PB, Berlin J, et al. Potentially curable pancreatic cancer: American Society of Clinical Oncology Clinical Practice Guideline. J Clin Oncol 2016;34:2541-56.

34. Balaban EP, Mangu PB, Khorana AA, et al. Locally advanced, unresectable pancreatic cancer: American Society of Clinical Oncology clinical practice guideline. J Clin Oncol 2016;34:2654-68.

35. Sohal DPS, Mangu PB, Khorana AA, et al. Metastatic pancreatic cancer: American Society of clinical oncology clinical practice guideline. J Clin Oncol 2016;34:2784-96.

36 Ducreux M, Cuhna AS, Caramella C, et al. ESMO Guidelines Committee. Cancer of the pancreas: ESMO Clinical Practice Guidelines for diagnosis, treatment and follow-up. Ann Oncol 2015;26(Suppl 5):v56-68.

37. Arnaoutakis GJ, Rangachari D, Laheru DA, et al. Pulmonary resection for isolated pancreatic adenocarcinoma metastasis: an analysis of outcomes and survival. Journal of Gastrointestinal Surgery 2011;15:1611-7.

38. Hwang JH, Kim JH, Lee JC. Neo tools for evidence mapping, PLOEM. Hwang-lab 2016. http://www.hwang-lab.com/03_ploem/

39. Lee JC, Kim HW, Hwang JH, et al. A novel modality for evidence mapping in systematic reviews: Plotting-E-Map (PLOEM). Cochrane Colloquium 2016. http://abstracts.cochrane.org/2016-seoul/novelmodality-evidence-mapping-systematic-reviews-plotting-e-mapploem

40. Moher D, et al. Preferred reporting items for systematic reviews and meta-analyses: the PRISMA statement. Ann Intern Med 2009;151:264-9.

41. Snilstveit B, Vojtkova M, Bhavsar A, et al. Evidence and gap maps: a tool for promoting evidence informed policy and strategic research agendas. J Clin Epidemiol 2016;79:120-9.

42. Bragge $P$, Clavisi $O$, Turner $T$, et al. The global evidence mapping initiative: scoping research in broad topic areas. BMC Med Res Methodol 2011;11:92.

43. Hetrick SE, Parker AG, Callahan P, et al. Evidence mapping: illustrating an emerging methodology to improve evidence-based practice in youth mental health. J Eval Clin Pract 2010;16:1025-30.

44. Katz DL, Williams AL, Girard C, et al. The evidence base for complementary and alternative medicine: methods of Evidence Mapping with application to CAM. Altern Ther Health Med 2003:9:22-30.

45. Mathes T, Pieper D. Clarifying the distinction between case series and cohort studies in systematic reviews of comparative studies: potential impact on body of evidence and workload. BMC Med Res Methodol 2017;17:107.

46. Dekkers OM, Egger M, Altman DG, et al. Distinguishing case series from cohort studies. Ann Intern Med 2012;156:37-40.

47. Higgins JPT, Altman DG, Gotzsche PC, et al. The Cochrane collaboration's tool for assessing risk of bias in randomised trials. BMJ 2011;343:d5928.

48. Sterne JAC, Hernán MA, Reeves BC, et al. ROBINS-I: a tool for assessing risk of bias in non-randomised studies of interventions. BMJ 2016;355:i4919.

49. Hoy D, Brooks P, Woolf $A$, et al. Assessing risk of bias in prevalence studies: modification of an existing tool and evidence of interrater agreement. J Clin Epidemiol 2012;65:934-9.

50. National Institute of Health. Quality assessment tool for case series studies. 2014. https://www.nhlbi.nih.gov/health-pro/guidelines/indevelop/cardiovascular-risk-reduction/tools/case series

51. Marshall Z, Welch V, Thomas J, et al. Documenting research with transgender and gender diverse people: protocol for an evidence map and thematic analysis. Syst Rev 2017;6:35 
52. Lunny C, Brennan SE, McDonald S, et al. Evidence map of studies evaluating methods for conducting, interpreting and reporting overviews of systematic reviews of interventions: rationale and design. Syst Rev 2016;5:4

53. Farah WH, Alsawas M, Mainou M, et al. Non-pharmacological treatment of depression: a systematic review and evidence map. Evid Based Med 2016;21:214-21.

54. Tierney JF, Stewart LA, Ghersi D, et al. Practical methods for incorporating summary time-to-event data into meta-analysis. Trials 2007;8:16.

55. DerSimonian R, Kacker R. Random-effects model for metaanalysis of clinical trials: an update. Contemp Clin Trials 2007;28:105-14.
56. Higgins JPT, Thompson SG. Quantifying heterogeneity in a metaanalysis. Stat Med 2002;21:1539-58.

57. Peters JL, Sutton AJ, Jones DR, et al. Contour-enhanced metaanalysis funnel plots help distinguish publication bias from other causes of asymmetry. J Clin Epidemiol 2008;61:991-6.

58. Cochrane Informatics \& Knowledge Management Department. GRADEpro 3.6 program. 2013. http://tech.cochrane.org/revman/ gradepro

59. Cochrane Community. RevMan 5 Software Program. Cochrane collaboration. 2015. http://community.cochrane.org/tools/reviewproduction-tools/revman-5

60. MRC Biostatics Unit. WinBUGS 1.4 Software Program. Univ. of Campridge, 2016. 